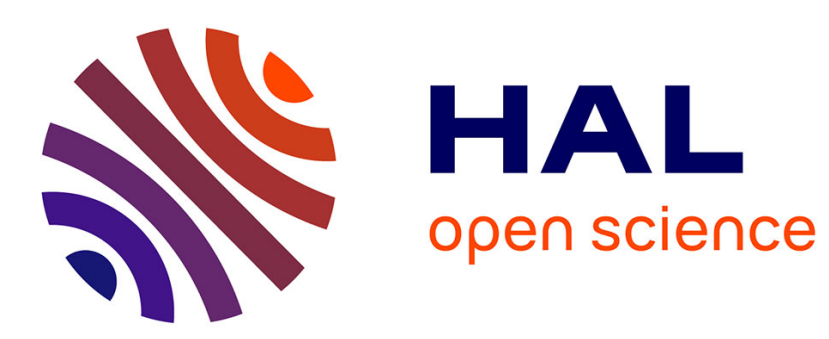

\title{
Adhesive Contact of Viscoelastic Spheres: a Hand-Waving Introduction
}

\author{
Etienne Barthel, Guillaume Haiat
}

\section{To cite this version:}

Etienne Barthel, Guillaume Haiat. Adhesive Contact of Viscoelastic Spheres: a Hand-Waving Introduction. Journal of Adhesion, 2004, 80 (1-2), pp.1-19. 10.1080/00218460490276722 . hal-00001437

\section{HAL Id: hal-00001437 \\ https://hal.science/hal-00001437}

Submitted on 9 Apr 2004

HAL is a multi-disciplinary open access archive for the deposit and dissemination of scientific research documents, whether they are published or not. The documents may come from teaching and research institutions in France or abroad, or from public or private research centers.
L'archive ouverte pluridisciplinaire HAL, est destinée au dépôt et à la diffusion de documents scientifiques de niveau recherche, publiés ou non, émanant des établissements d'enseignement et de recherche français ou étrangers, des laboratoires publics ou privés. 


\title{
ADHESIVE CONTACT OF VISCOELASTIC SPHERES:
}

\section{A HAND-WAVING INTRODUCTION}

\section{E. Barthel ${ }^{l}$ and G. Haiat ${ }^{2}$,}

1) Surface du Verre et Interfaces, CNRS/Saint-Gobain, Aubervilliers, France,

2) CEA, Laboratoire de Simulation Ultrasonore et de Traitement, Gif-sur-Yvette, France.

etienne.barthel@saint-gobain.com

\begin{abstract}
:
We give an overview of the general features of the linear viscoelastic adhesive contact model. The two main features are 1) a delay between the contraction of the contact radius and the onset of the indenter retraction; 2) the enhancement of the adherence force. We emphasize the role played by stress relaxation within the contact zone in these phenomena and give simple forms of the viscoelastic adhesive contact equations to account for it. Two characteristic timescales are identified, respectively associated with the crack tip and the contact zone. Their asymmetric roles in the growing and receding contact phases is evidenced. Energy release rates for both phases are calculated together with their irreversible components.
\end{abstract}

Keywords: adhesion, adherence, contact mechanics, linear viscoelasticity, viscoelastic crack propagation.

\section{Introduction}

Probing the adherence of soft viscoelastic solids, as in the JKR test [1,2], assessing the mechanical properties of polymers in small scale contact experiments like nanoindentation [3] 
or AFM [4-6], where surface forces interfere, or understanding the adhesion of molten glass to hot molds all require a model for the adhesive contact of viscoelastic bodies.

Sometime ago, in this same journal, we had shown that the Sneddon method, based on the systematic application of Hankel transforms, provides wide reaching insight into the elastic contact problems of axisymmetric bodies [7]. Relying on the same method, we have recently proposed a theory of the adhesive contact within the linear viscoelastic regime $[8,9]$. The aim of the present contribution is to parallel our previous paper on the elastic case [7] with an exposition of the main ideas behind the adhesive contact of viscoelastic bodies.

Let us recall the main steps in the development of the viscoelastic adhesive contact theory; a more comprehensive bibliographical list may be found in $[8,9]$.

In the $60 \mathrm{~s}^{\prime}$, the viscoelastic adhesionless contact problem has spurred a number of efforts, finally yielding Ting's completely satisfactory theory in 1966 [10]. The next step, in the 70s', was taken by Schapery, who described crack propagation in a viscoelastic medium by embedding a process zone into a linear elastic solid [11-14]. Coupling a viscoelastic crack behavior with an elastic contact has provided a first category of viscoelastic adhesive contact models [15]. One further step was taken when Hui et al. coupled a viscoelastic contact model to a viscoelastic crack model [16], as initially suggested by Schapery [14]. Their theory, however, is valid for an increasing contact radius only. Their attempt for a decreasing contact radius proved less successful $[17,18]$.

The model for the viscoelastic adhesive contact we have recently proposed [8] is based on the Sneddon method of Hankel transform [7]. In a suitable limit [9], it turns out to simply couple Ting's model for the adhesionless viscoelastic contact and Schapery's viscoelastic crack approach. Under this form, it takes a particularly simple structure, in close connection with the JKR model [19] for elastic adhesive contacts. The aim of the present paper is to highlight 
this connection and to present the main concepts underlying the adhesive linear viscoelastic contact model.

\section{Description of the adhesive contact}

The physics of the contact between two bodies is subtle and has to be simplified to be efficiently accounted for in a mechanical model. Several paths [20] may be followed for that purpose: one of them is to assume infinite repulsion at contact; and before contact, attractive interaction between surfaces over some finite range (Fig. 1). Then, in the adhesive contact, we can identify two zones: in the contact zone, the surfaces touch each other; outside this zone, in the interaction zone, tensile stresses are present without contact (Fig. 2).

We now examine the consequence of these assumptions on the mechanics of the contact.

\subsection{Boundary conditions}

\subsubsection{The inner problem: contact variables}

Inside the contact zone, the fact that the surfaces come to contact is specified by the following boundary conditions:

$u(t, r)=\delta(t)-f(r)$ for $\quad r<a(t)$

where $u(t, r)$ is the normal surface displacement, $\delta(t)$ the penetration, $f(r)$ the shape of the indenter and $a(t)$ the contact radius. The main variables for the contact problem itself are thus the penetration and the contact radius. The third contact variable, the force, although often directly measured in practice, plays a less direct role in the theory, because it is the integral of the surface stress distribution and therefore specifies the boundary conditions less directly.

\subsubsection{The outer problem: the interaction zone variables}

Adhesion is expressed by the following boundary conditions:

$$
\left\{\begin{array}{c}
\sigma(r)=-p(r) \text { for } a<r<(a+\varepsilon) \\
\sigma(r)=0 \text { for }(a+\varepsilon)<r
\end{array}\right.
$$


where $p(r)$ is a stress distribution relevant to the physics of the adhesive process, $\sigma(r)$ is the normal surface stress, and $\varepsilon$ the size of the interaction zone (Fig. 2).

\subsection{Self-consistent description of the interaction zone}

In the interaction zone, normal surface stress, deformation and interactions are intimately coupled: the normal surface stress is a function of the of the gap between surfaces (Fig. 1), which itself depends upon the surface deformation, which is controlled by the normal surface stresses. As a result, a self consistent treatment is required $[7,11]$. The final useful equation is typically of the form

$$
w=\int_{a}^{\infty} d r \sigma(r) \frac{d h(r)}{d r}
$$

where $w$ is the adhesion energy and $h(r)$ the gap between surfaces. Although we more or less implicitly assume an interaction potential here, there is a priori no limitation to generalizing this method to more complex adhesive phenomena.

The difficult issue here is that the mechanical relation between stress and surface displacement (and therefore the gap $h(r)$ ) is non local, so that explicit expressions for (5) are often intricate. This treatment is simplified if we assume that the contribution of the interaction zone surface stresses to the interaction zone surface deformation dominates the contribution of contact zone stresses. An equivalent assumption is that the interaction zone size $\varepsilon$ is much smaller than the contact radius $a$. This is the essence of the JKR limit [19]. Then, the gap shape is dominated by the adhesive stress induced deformations [7], and equation (3) reduces to

$$
w \propto \frac{\sigma^{2} \varepsilon}{E^{*}}
$$

in which $E^{*}$ is the reduced modulus defined by (A4) in the appendix and the proportionality coefficient depends upon the details of the interaction. Under this form, the form of an elastic 
energy release rate, the self-consistency equation (4) lends itself to a linear elastic fracture mechanics interpretation. For that purpose, in our formalism, we introduce a new quantity, $g(a)$, which, as will be shown below, naturally couples the contact and the interaction zones.

On the interaction zone side, $g(a)$, which is defined in the appendix by (A1), is a function of the interaction stress distribution $p(r)$ (as defined in (2)) only. We have also shown previously [7] that in the elastic case, (4) can be written

$w=\frac{2 g(a)^{2}}{\pi E^{*} a}$

Therefore, $g(a)$ assumes the status of a stress intensity factor. Indeed, denoting $K$ the interaction zone stress intensity factor at $a$, we have shown [9] that

$$
K=\frac{-2 g(a)}{\sqrt{\pi a}}
$$

Thus, the self consistent treatment of the interaction zone essentially specifies the stress intensity factor, $K$ or $g(a)$, characteristic of the adhesive interaction stress distribution. We now discuss how $g(a)$ determines the contact variables.

\subsection{Coupling the interaction zone to the contact zone}

If the adhesive interaction is zero, then the solution to the contact problem is the Hertz theory [21] for a spherical indenter (and its extensions for other geometries), which specifies the penetration $\delta$ as a function of the contact radius $a$. This function $\delta_{0}(a)$ depends upon the shape of the indenter $f(r)$ only. A general approach to the adhesive contact problem is then to specify the adhesive process and solve the interaction zone problem. The actual attractive stress distribution is thus determined. However, this attractive stress distribution pulls on the surfaces and, for a given penetration, increases the contact radius. The penetration is then 


$$
\delta(t)=\delta_{0}(a)+\frac{2}{E^{*}} g(a)
$$

This equation shows that for a given contact radius, the additional term for the penetration is proportional to the adhesive interaction stress intensity factor, or more directly to $g(a)$. Note that $g(a)$ is negative, so that, for a given contact radius, a reduction of the penetration with adhesion is predicted by (7).

Another interpretation of (7) stems from the observation that the quantity $\frac{\sqrt{\pi a} E^{*}}{4}\left(\delta-\delta_{0}\right)$ is the stress intensity factor generated by the additional stress distribution due to the additional flat punch displacement $\left(\delta-\delta_{0}\right)$. Then, (7) states that this stress singularity is cancelled by the stress singularity due to the outer attractive stress distribution [22-23].

The penetration equation (7) and the force equation [7], which can be derived from (A9) in the appendix, form the contact equations which, together with the self consistency equation (5), provide the solution to the linear elastic adhesive contact problem.

\section{Viscoelastic Contact: main results}

We are now in a position to extend the previous model to viscoelastic behaviour by assuming a delayed elastic behaviour. We introduce the usual viscoelastic creep $\varphi$ and relaxation $\psi$ functions (Fig. 3). Stress $\sigma$ and deformation $\varepsilon$ now obey

$$
\sigma(t)=\int_{0}^{t} d \tau \psi(t-\tau) \frac{d \varepsilon}{d \tau}
$$

and

$$
\varepsilon(t)=\int_{0}^{t} d \tau \varphi(t-\tau) \frac{d \sigma}{d \tau}
$$

Under suitable conditions [8], this results in the description of the mechanics in terms of reduced creep $\varphi^{*}$ and relaxation $\psi^{*}$ functions. 
As mentioned previously, in section 2.2, it is usually reasonable to assume that the interaction zone problem is local to the crack tip $(\varepsilon<a)$. Then contact zone and interaction zone are coupled only through the variable $g(a)$. Under this assumption, we first consider the viscoelastic crack propagation.

\subsection{Self consistent crack problem}

The adhesive viscoelastic problem also requires some details of the physical process giving rise to adhesion. In the present approach, we suppose a "double-Hertz" interaction zone with characteristic stress $\sigma_{0}$ and adhesion energy $w$ [24]. This model is similar to a Dugdale model [22].

We have shown [9] that

$g(a)=-\frac{\pi}{4} \sigma_{0} \sqrt{2 a \varepsilon}$

In the viscoelastic case, time now plays a role so that a local timescale appears: $t_{r}$, the time required by the crack to move a distance equal to the interaction zone size $\varepsilon$ (Fig. 2). As a result, we have a relation between the crack velocity (or contact radius velocity) $d a / d t, \varepsilon$ and $t_{r}$ :

$\frac{d a}{d t}=\frac{\varepsilon}{t_{r}}$

Then, we obtain [9] that the viscoelastic crack behavior is given by:

$$
w=\frac{2 g(a)^{2}}{\pi a} \varphi_{1}^{*}\left(t_{r}\right)
$$

where

$$
\varphi_{1>}^{*}(t)=\frac{2}{t^{2}} \int_{0}^{t}(t-\tau) \varphi_{1}^{*}(t-\tau) d \tau
$$

when the contact radius increases and 


$$
\varphi_{1<}^{*}(t)=\frac{2}{t^{2}} \int_{0}^{t} \tau \varphi_{1}^{*}(t-\tau) d \tau
$$

when the contact radius decreases.

We note that the form of (10) is identical to the form of (5), but the stress intensity factor is calculated from an effective compliance $\varphi_{1}^{*}\left(t_{r}\right)$, which depends upon the crack tip velocity. This effective compliance amounts to the instantaneous compliance when $t_{r}$ is zero, is the long time compliance when $t_{r}$ is infinite, and lies in between for intermediate $t_{r}$.

These results, which are arrived at through the treatment of the full contact problem [9], are comparable to Schapery's viscoelastic crack propagation models.

From (10-12), the stress intensity factor of the attractive interaction stress distribution can be calculated as a function of crack tip velocity. The typical behavior is exemplified in Fig. 4. This stress intensity factor has been identified above as the key parameter in the determination of the penetration, as we now discuss in more details.

\subsection{Penetration}

We now couple the viscoelastic crack problem to the viscoelastic contact problem.

\subsubsection{Inward (closing crack)}

We obtain [9] for increasing contact radius

$\delta(t)=\delta_{0}(a(t))+2 g(a(t)) \varphi_{0}^{*}\left(t_{r}\right)$

where

$\varphi_{0}^{*}(t)=\frac{1}{t} \int_{0}^{t} \varphi^{*}(t-\tau) d \tau$

This penetration equation is equivalent to the elastic case (Eq. (7)) provided the effective compliance $\varphi_{0}^{*}\left(t_{r}\right)$ is substituted to the elastic compliance $1 / E^{*}$. Setting $g=0$, we recover the adhesionless viscoelastic case 
$\delta(t)=\delta_{0}(a(t))$

Note that $\varphi_{0}^{*}(t)$ is larger than $\varphi^{*}(0)$ : due to creep, the penetration correction is larger than in the elastic case.

\subsubsection{Outward (opening crack)}

For decreasing contact radius, the main term is [9]

$\frac{1}{2} \int_{t_{-}}^{t} \psi^{*}(t-\tau) \frac{d \delta}{d \tau} d \tau=g(a(t))$

A corrective term which is not essential to understand the physics of the adhesive contact has not been included here. The time $t_{-}$is the time at which the present contact radius $a(t)$ was met during the increasing contact radius phase. Once again, setting $g=0$, we recover the adhesionless solution by Ting [10]

$0=\int_{t}^{t} \psi^{*}(t-\tau) \frac{d \delta}{d \tau} d \tau$

Equation (16) is central to the viscoelastic contact rupture. Comparing with (13), we observe that its structure is exactly inverse. The right-hand side is proportional to the attractive interaction stress intensity factor. But the viscoelastic effect, instead of scaling the stress intensity factor with the creep function and the local timescale $t_{r}$, now appears under the form of a convolution of the penetration with the relaxation function over the full history of the system - that is to say between $t_{-}$and $t$.

This form of the penetration equation is best explained if the cancellation of inner and outer stress intensity factors formulation $(\S 2.3)$ is retained. This formulation gives to the left hand side in (16) the meaning of an inner stress intensity factor at $t$ and $a(t)$, which results from the flat punch displacement $\delta(t)$ convoluted by the stress relaxation function $\psi$. 


\subsection{Force}

Although the force is a less direct expression of the contact boundary conditions, it is useful in practice because it is most readily obtained experimentally.

Introducing $f(\delta, a)=a \delta-\int_{0}^{a} d r \delta_{0}(r)$, where the second term depends only upon the shape of the indenting body, from (A9) in the appendix, we have:

a) for adhesionless elastic contact: $F_{0}(a)=2 E^{*} f_{0}(a) \equiv 2 E^{*} f\left(\delta_{0}(a), a\right)$;

b) for an adhesive elastic contact: $F(a)=F_{0}(a)+4 \operatorname{ag}(a)$.

\subsubsection{Inward}

In the viscoelastic case, in the increasing contact radius case, the force is

$$
F(t)=2 \int_{0}^{t} \psi^{*}(t-\tau) \frac{d f(\delta(\tau), a(\tau))}{d \tau} d \tau
$$

from which the stress intensity factor may be extracted as

$$
g(a)=\frac{\int_{0}^{t} \varphi^{*}(t-\tau) \frac{\partial F(\tau)}{\partial \tau} d \tau-\varphi^{*}(0) F_{0}(a)}{4 a \varphi_{0}^{*}\left(t_{r}\right)}
$$

From this equation and the self-consistency equation (10), $d a / d t$ can be extracted, so that by integration $a(t)$, and ultimately $\delta(t)$ are known.

For instance, penetration under vanishing external force entails $f(\delta(t), a(t))=0$. Therefore, $\delta(t)=a^{2} / 3 R$

\subsubsection{Outward}

The force is

$$
F=2 \int_{0}^{t_{-}} \psi^{*}(t-\tau) \frac{d f(\delta(\tau), a(\tau))}{d \tau} d \tau+4 \operatorname{ag}(a)
$$

from which $g(a)$ is directly extracted. Here again, the adhesionless case is readily obtained. 


\subsection{The adhesive viscoelastic contact: main phenomena}

The two main phenomena which signal viscoelastic behaviour in the adhesive contact will now be explained briefly.

\subsubsection{The stick zone}

The first characteristics of the viscoelastic adhesive contact is the delay between the time when the indenter starts moving backwards (Fig. 5) and the time when the contact radius starts to recede markedly. This delay we called the stick time [9] (Fig. 6). It is due to the fact that, in the region where the contact radius is maximum, the contact radius velocity is close to zero. Then, the interaction stress intensity factor, and $g(a)$, are small (Fig. 4). To get significant propagation, we must restore a higher $|g(a)|$. This is obtained by the backward motion of the indenter, but the effect is qualified by the stress relaxation (16). The condition for propagation is achieved only when the right hand side member in (16) is large enough, i.e. when the backward motion of the indenter overcomes the stress relaxation. This is the origin of the stick time.

\subsubsection{Adherence force enhancement}

The pull-out force in the elastic adhesive contact, in the small interaction zone size (or JKR) limit is $3 / 2 \pi R w$. Its independence from the actual elastic modulus of the contacting bodies is most noteworthy. It is due to the fact that compressive and tensile stresses within the contact zone balance each other at pull-out.

For viscoelastic bodies, however, the picture is quite different (Fig. 7). Restoring a large stress intensity factor by the motion of the indenter $\delta(t)$ brings back a sizeable tensile flat punch stress distribution within the contact zone (Fig. 8). At the same time, the compressive stresses within the contact zone have also decayed but are not restored by the present motion. This is apparent for instance in (23) where the first (compressive) term decays as $\psi^{*}(t)$ while the 
second (tensile), originating from the flat punch tensile stress distribution, is identical in the elastic case.

Once again neglecting corrective terms in the increasing contact radius part of the contact, (23) may be written simply

$$
F=\psi^{*}(t) f_{0}(a(t))+4 a g(a(t))
$$

We conclude that the decay of the compressive stress distribution within the contact zone, and therefore of its contribution to the total force, leads to the enhancement of the overall adhesive force (Fig. 8).

\subsection{Energy}

\subsubsection{Energy release rate}

The energy release rate, which is the mechanical energy expended in propagating the contact per unit area, is expressed from (A10) in the appendix, as

$G=\frac{d \Omega}{d A}=\frac{1}{\pi a} g(a(t), t) \theta(a(t), t)$

where the quantity $\theta(a, t)=\delta(t)-\delta_{0}(a)$ is closely connected with the penetration equations (7), (13) and (16). $G$ is zero in the absence of adhesion. If the contact is elastic, (5), (7) and (25) show that $G=w$. Since $w$ is the adhesion energy, gained from the crack propagation, this equality means reversible propagation.

In the viscoelastic case, let us denote $G_{>}$the energy release rate for increasing contact radius. Equation (13) results in

$G_{>}=\frac{2 g(a(t))^{2} \varphi_{0}^{*}\left(t_{r}\right)}{\pi a}$

Comparison with (10) shows that for increasing contact radius (closing crack),

$\frac{G_{>}}{w}=\frac{\varphi_{0}^{*}\left(t_{r}\right)}{\varphi_{1>}^{*}\left(t_{r}\right)} \leq 1$ 
Mathematically, the $G_{>} / w$ ratio is smaller than one because $\varphi^{*}$ is a monotonic increasing function. Physically, it means that the propagation of the crack is dissipative. However, we note that equality holds when the crack is very fast and also when it is very slow: the system is then effectively elastic. Reversibility of the crack propagation at high velocity is at variance with results by Schapery [11,12] and subsequently Greenwood and Johnson [13]. The reason is that Schapery assumes the relaxed state as the reference state. With fast cracks, however, this relaxed reference state is reached nowhere near the crack tip. Our present estimate of the dissipation is purely local, at the crack tip; dissipation due to stress relaxation inside the contact itself, which is also present in the adhesionless contact, is not included in the present expression for $G_{>}$.

A similar discussion for the receding contact radius phase is less straightforward because (16), however approximate, takes into account the full history of the system. We will therefore provide an approximate discussion, restricted to a special case. We assume that (Fig. 5)

1) loading to the maximum penetration $\delta_{m}$ is fast;

2) unloading takes place immediately after loading;

3) the unloading rate $d \delta / d t$ is constant.

Then, (16) becomes:

$g(a(t))=\frac{\psi(t)}{2}\left(\delta_{m}-\delta_{0}(a(t))\right)+\frac{\psi_{0}^{*}(t)}{2}\left(\delta(t)-\delta_{m}\right)$

where

$\psi_{0}^{*}(t)=\frac{1}{t} \int_{0}^{t} \psi^{*}(t-\tau) d \tau$

Now $0<\psi^{*}(t)<\psi_{0}^{*}(t)$, so that, since $g(a(t), t)$ is negative, we have

$2 g(a(t)) / \psi^{*}(t)<2 g(a(t)) / \psi_{0}^{*}(t)<\theta(a(t), t)<0$

and $\theta(a(t), t)$ is also negative 
$G_{<}>\frac{2 g(a(t))^{2}}{\pi a \psi^{*}(t)}$

As a result, in the decreasing contact radius phase (growing crack)

$\frac{G_{<}}{w} \geq \frac{1}{\psi^{*}(t) \varphi_{1<}^{*}\left(t_{r}\right)}$

Typically, we may expect the experimental time $t$ to be large and $t_{r}$ to be small, at least when the contact recedes markedly. Then $\psi_{0}^{*}(t)$ is of the order of the relaxed modulus $\psi^{*}(+\infty)$ : Schapery's relaxed reference state is recovered. Simultaneously, $\varphi_{1<}^{*}\left(t_{r}\right)$ is of the order of the instantaneous compliance $1 / \psi^{*}(0)$, and [15]

$\frac{1}{\psi^{*}(t) \varphi_{1<}^{*}\left(t_{r}\right)} \propto \frac{\psi^{*}(0)}{\psi^{*}(+\infty)}$

which is much larger than 1 for a significantly viscoelastic material: a large dissipation appears in the outward phase.

Then $G_{<} \geq w$, but equality is restored at slow velocities (for a finite long time compliance) or for a loading cycle faster than any typical relaxation time.

This dissipation can be rationalized in the following manner: the crack tip, which moves fast, with characteristic time $t_{r}$, feels an effectively harder material (10). The flat punch displacement, however, applies to an effectively softer material, because of the viscoelastic stress relaxation (28) applies to the characteristic time $t$. As a result, for the same stress intensity factor, the flat punch energy release rate is much larger than the crack energy release rate. The energy difference is dissipated.

\section{Discussion}

Within the contact zone, we find both compressive (at the center) and tensile stresses (at the periphery). The normalized 1.5 adherence force for an elastic adhesive (JKR [19]) contact 
results from a balance between these two stress contribution. For viscoelastic bodies, however, the stress distribution inside the contact zone relaxes. As a result, the contact zone does not recede as soon as the indenter is pulled back, because the stress intensity factor is low, which leads to low contact radius velocities (Fig. 4). One requires sufficient (and sufficiently fast) backward motion of the indenter to restore a stress intensity factor large enough for the contact radius to actually decrease (Eq. 16).

However, this additional tensile stress distribution (a flat-punch like stress distribution) does not contribute compressive stresses. Consequently, the balance between compressive and tensile stresses one finds in the elastic case is now offset, resulting in an enhanced adherence force.

\section{Conclusion}

Two phenomena must be included in a complete model for the adhesive contact of viscoelastic spheres. Creep in the interaction zone reduces the stress intensity factor though a larger effective compliance. At the same time, stress relaxation inside the contact zone induces both a time lag between indenter retraction and contact radius decrease ("stick" effect) and an enhancement of the adherence force through unbalance between compressive and tensile stresses. Our models [8,9] provides a complete description of this combination of phenomena.

\section{References}

[1] Falsafi A., Tirrell M. and Pocius A., Langmuir 16, 1816 - 24 (2000).

[2] Crosby A. J., Shull K. R., Lin Y. Y. and Hui C.-Y., J. Rheol. 46, 273 - 94 (2002).

[3] Giri M., Bousfield D. B. and Unertl W. N., Langmuir 17, 2973 - 81 (2001).

[4] Basire C. and Frétigny C., Tribology Letters 10, 189 - 93 (2001). 
[5] Portigliatti M., Koutsos V., Hervet H. and Leger L., Langmuir 16, 6374 - 76 (2000).

[6] Pickering J. P. and Vancso G. J., J. Macromol. Symp., 166, 189 - 99 (2001).

[7] Huguet A.-S. and Barthel E., J. Adhesion 74, 143 - 75 (2000).

[8] Haiat G., Phan Huy M.-C. and Barthel E., J. Mech. Phys. Solids 51, 69 - 99 (2003).

[9] Barthel E. and Haiat G., Langmuir 18, 9362 - 70 (2002).

[10] Ting T. C. T., J. Appl. Mech. 33, 845 - 54 (1966).

[11] Schapery R. A., Int. J. Fract. 11, 141 - 59 (1975).

[12] Schapery R. A, Int. J. Fract. 11, 369 - 88 (1975).

[13] Greenwood J. A. and Johnson K. L., Philos. Mag. A 43, 697 - 711 (1981).

[14] Schapery R. A., Int. J. Fract. 39, 163 - 189 (1989).

[15] Johnson K. L. in Tsukruk, V. V., Wahl, K. J. (Eds.), Microstructure and Microtribology of Polymer Surfaces, ACS, Washington, D.C., (1999).

[16] Hui C. H., Baney J. M. and Kramer E. J., Langmuir 14, 6570 - 78 (1998).

[17] Lin Y. Y., Hui C. Y., and Baney J. M., Journal of physics D, Applied physics 32, 2250 60 (1999).

[18] Lin Y. Y. and Hui C. Y., J. Polymer Sci. B, 40, 772 - 93 (2002).

[19] Johnson K. L., Kendall K. and Roberts A. D., Proc. Roy. Soc. London A 324, 301 - 13 (1971).

[20] Barthel, E., J. Colloid Inter. Sci. 200, 7 - 18 (1998).

[21] Hertz H., J. Reine Angew. Math. 92, 156 -171 (1882).

[22] Maugis D., J. Colloid Inter. Sci. 150, 243 - 69 (1992).

[23] Maugis D. and Barquins M., J. Phys. D:Appl. Phys., 11, 1989 - 2023 (1978).

[24] Greenwood J. A. and Johnson K. L., J. Phys. D: Appl. Phys. 31, 3279 (1998).

[25] Sneddon I. N., Fourier Transforms, McGraw Hill: New York (1951). 


\section{Captions:}

Fig. 1: Typical dependence of the interaction stresses with the gap (or distance) between surfaces, as assumed in the present contact model.

Fig. 2: Left: contact zone (radius $a$ ) and interaction zone (size $\varepsilon$ ) in a typical adhesive contact. Right: definition of the dwell time $t_{r}$.

Fig. 3: Typical time dependence of the viscoelastic stress relaxation $\psi$ and creep $\varphi$ functions.

Fig. 4: Typical stress intensity factor $K$ or $g(a)$ dependence upon contact radius velocity. $K$ decreases with velocity because the materials is effectively softer at lower velocity.

Fig. 5: Typical penetration history for an adhesive contact experiment.

Fig. 6: Typical contact radius history for a penetration history as in Fig. 5: most prominent is the so-called stick phase, where the contact radius stays close to constant while the penetration decreases.

Fig. 7: Typical behavior of force as function of time: elastic (dashed) and viscoelastic (full) for the penetration history in Fig. 5. The prominent feature is the enhancement of the adherence force mainly due to stress relaxation within the contact zone.

Fig. 8: Typical stress distribution in an adhesive contact (c): it is the linear superposition of the compressive adhesionless contact stress distribution for a penetration $\delta_{0}(a)$ (shown here for a sphere) (a) and the tensile flat punch distribution (b). The stress singularity in (b) is proportional to the displacement correction $\delta-\delta_{0}(a)$. In the viscoelastic contact, the contact time $t$ controls the amplitude of the compressive stress distribution through the stress relaxation function. A reduced compressive stress distribution leads to an enhanced adherence force. 


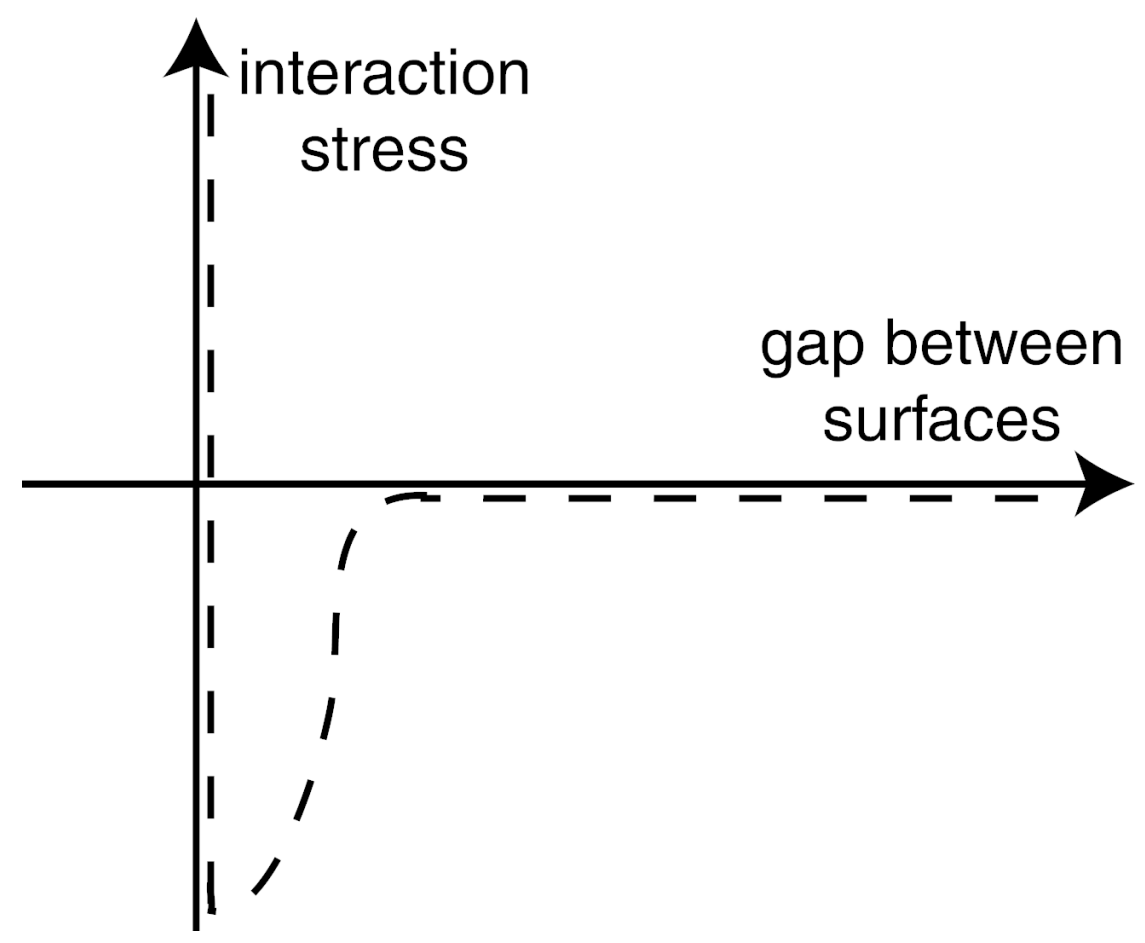

Barthel, Haiat, Fig. 1 


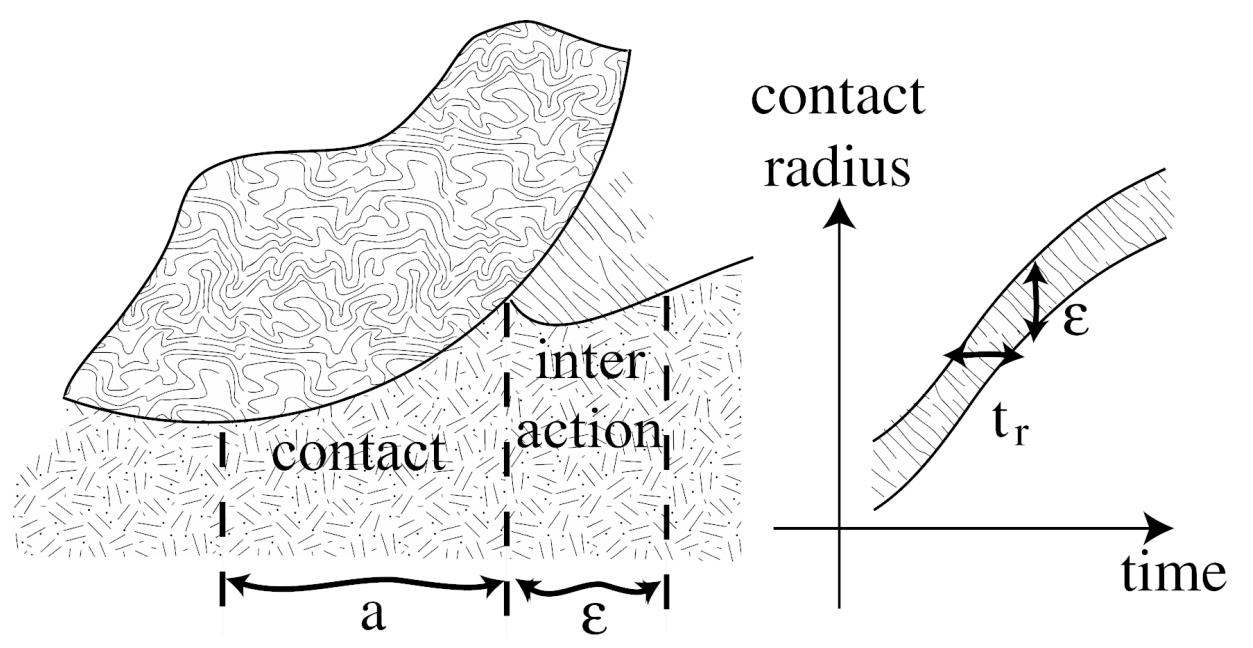

Barthel, Haiat, Fig. 2 


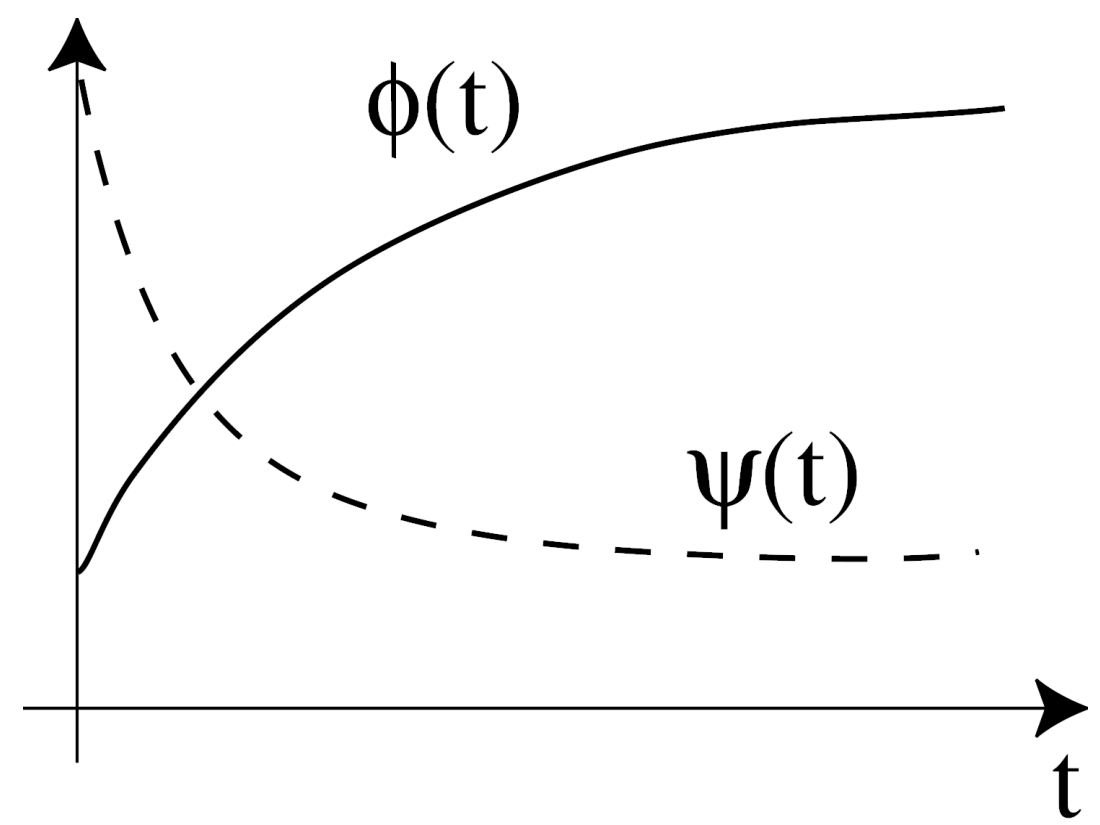

Barthel, Haiat, Fig. 3 


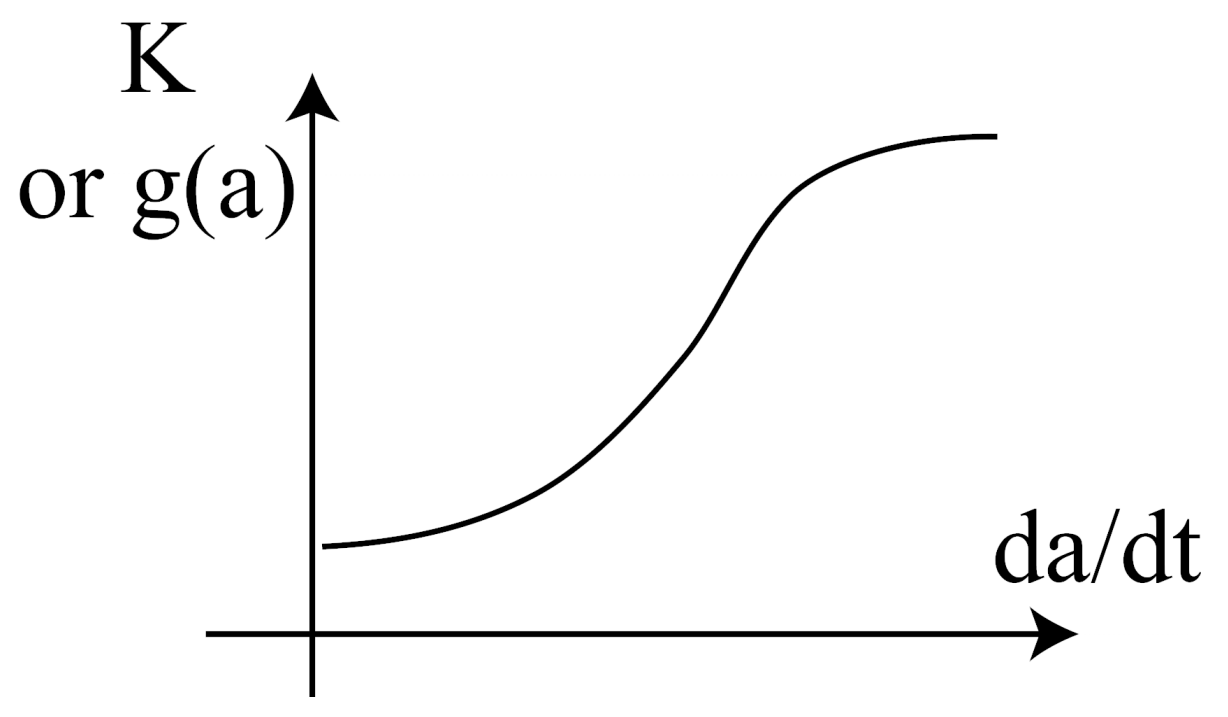

Barthel, Haiat, Fig. 4 


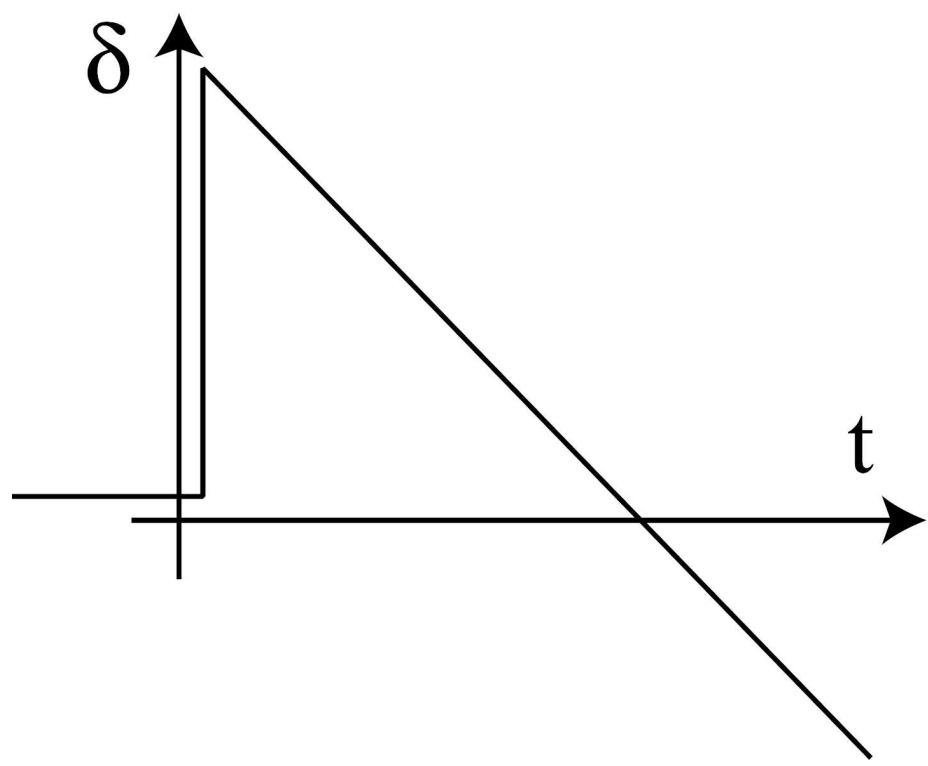

Barthel, Haiat, Fig. 5 


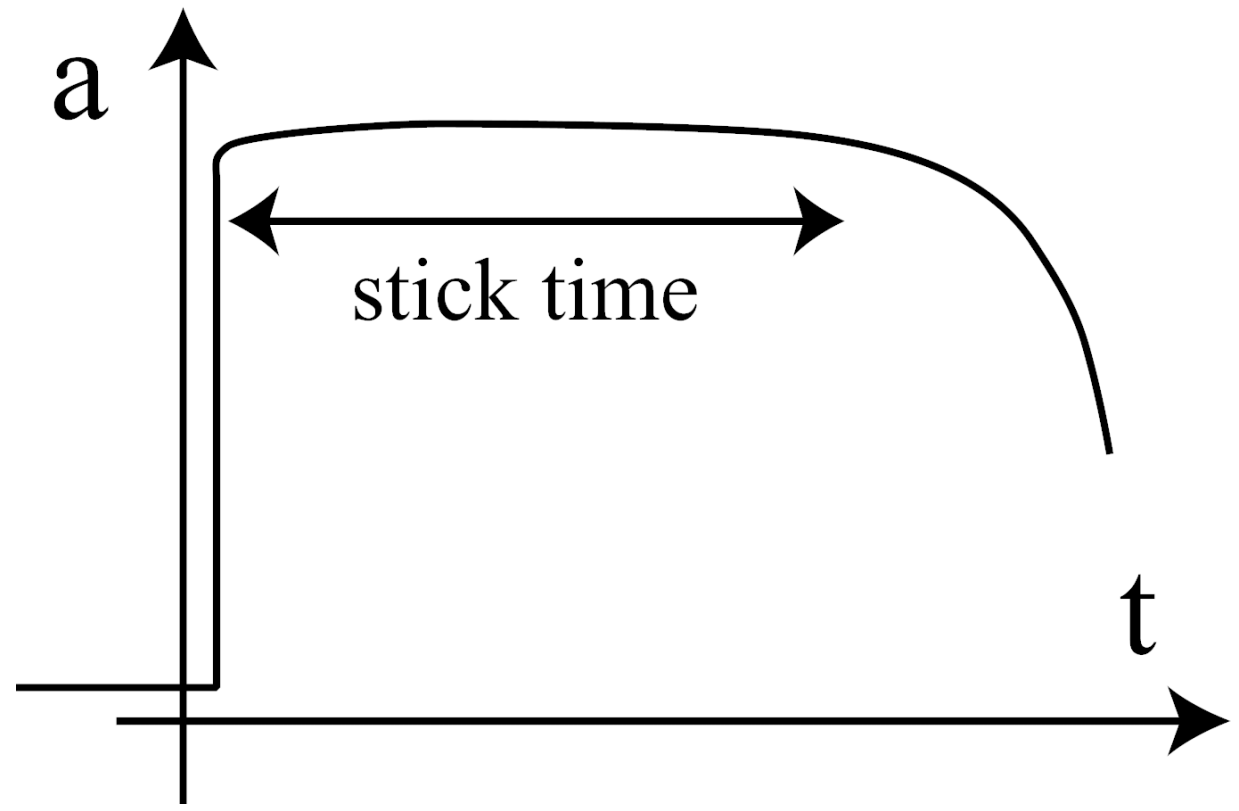

Barthel, Haiat, Fig. 6 


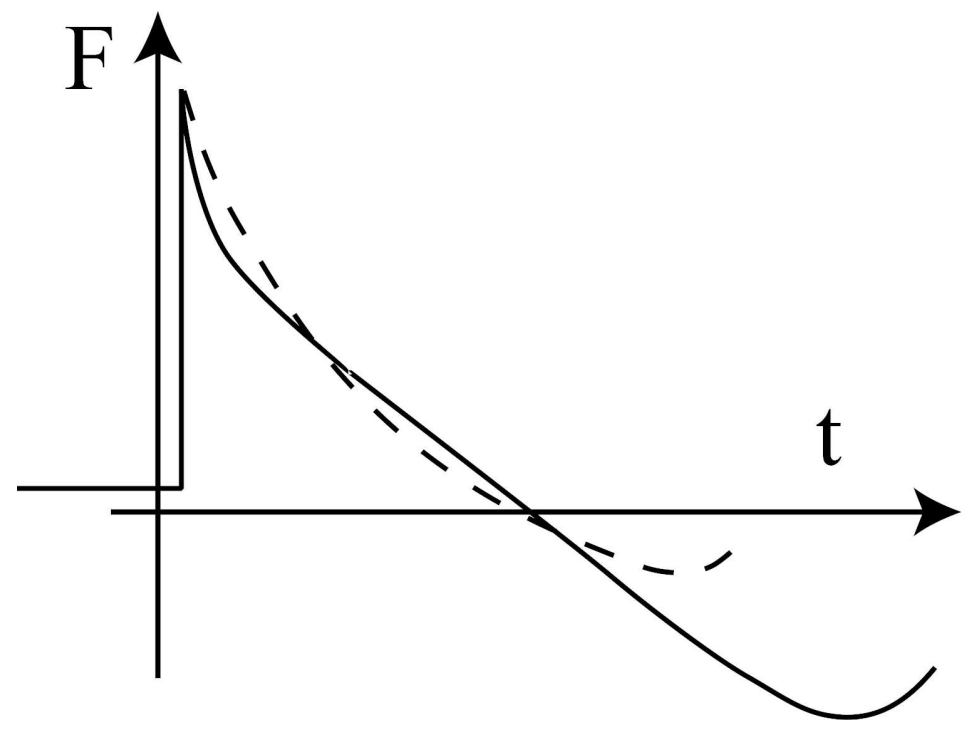

Barthel, Haiat Fig. 7 


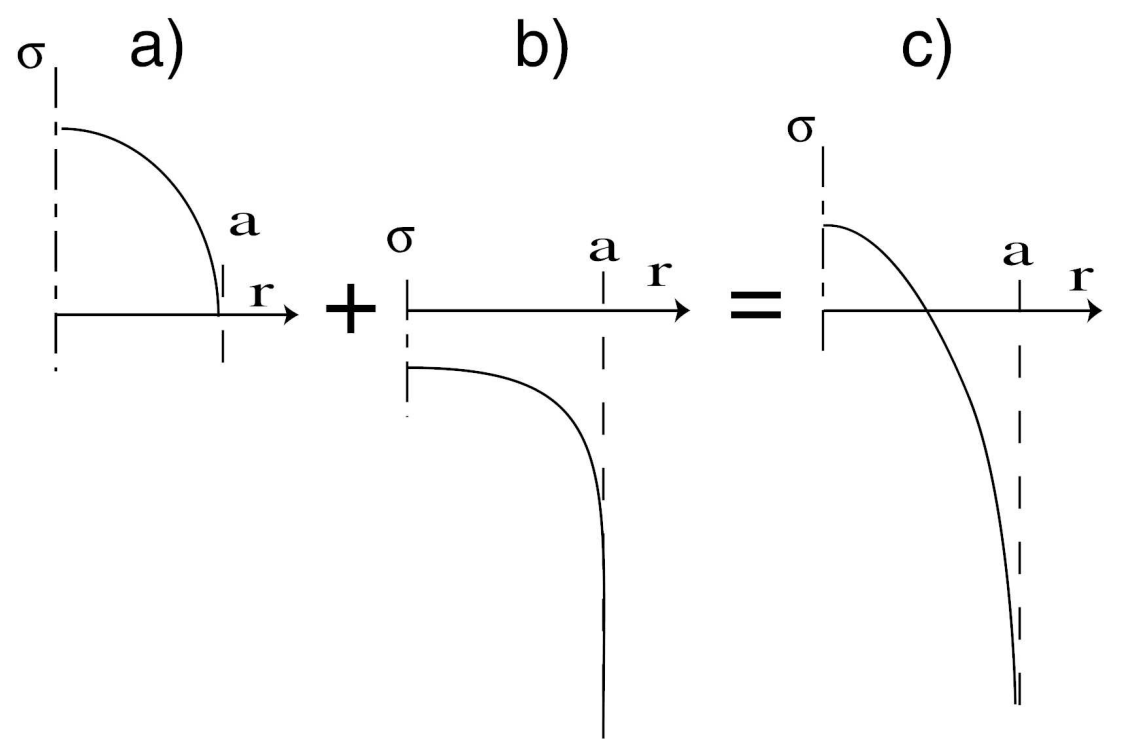

Barthel, Haiat, Fig. 8 


\section{Appendix: Surface Elasticity: linear viscoelastic case}

\subsection{Equilibrium}

Our usual method is to resort to specific transforms of the surface normal stress $\sigma(r)$ and surface normal displacement $u(r)$ distributions, as suggested by Sneddon [25]. These transforms are:

$$
\begin{aligned}
& g(r)=-\int_{r}^{+\infty} \frac{s \sigma(s)}{\sqrt{s^{2}-r^{2}}} d s \\
& \theta(r)=\frac{d}{d r} \int_{0}^{r} \frac{s u(s)}{\sqrt{r^{2}-s^{2}}} d s
\end{aligned}
$$

They are easily expressed in terms of the boundary conditions and simultaneously result in a local equilibrium equation:

$g(r)=\frac{E^{*}}{2} \theta(r)$

where the elastic surface compliance

$E^{*}=\frac{E}{1-v^{2}}$

(Young's modulus $E$ and Poisson ratio $v)^{1}$.

This approach contrasts with the direct method in the sense that the relation between surface stress and surface penetration

\footnotetext{
${ }^{1}$ Note that in contrast to our previous papers, we will here use the contact mechanics standard definition $E^{*}$ for the reduced modulus instead of $K=E^{*} / 2$. Similarly, we will use the notation $\psi^{*}=2 \psi$ and $\varphi^{*}=2 \varphi$, where $\psi$ and $\varphi$ were the reduced stress and relaxation functions in our previous papers. The ${ }^{*}$ notation is used throughout the paper to denote such reduced quantities, not dynamic material properties.
}

Document 1 $-26-$ 
$u(s)=\frac{1}{\pi E^{*}} \frac{p(r)}{|r-s|}$

has now been diagonalized.

Boundary conditions determine

$\theta(t, r)=\delta(t)-\delta_{0}(r)$ for $r<a(t)$

In addition, it is easily generalized to the linear viscoelastic case. Following the standard treatment of linear viscoelasticity as delayed elasticity, we introduce the reduced creep function $\varphi^{*}(t)$ and relaxation function $\psi^{*}(t)$. Then, the equilibrium equation for a viscoelastic contact becomes

$g(r, t)=\int_{0}^{t} \psi^{*}(t-\tau) \frac{d \theta(r, \tau)}{d \tau} d \tau$

or inversely

$\theta(r, t)=\int_{0}^{t} \varphi^{*}(t-\tau) \frac{d g(r, \tau)}{d \tau} d \tau$

\subsection{Force and Energy Stored}

With these notations, we obtain simple expressions for the total force

$F=4 \int_{0}^{+\infty} g(r) d r$

and total elastic energy stored

$\Omega=2 \int_{0}^{+\infty} g(r) \theta(r) d r$ 